\title{
Dynamic changes in the gene expression profile during rat oral carcinogenesis induced by 4-nitroquinoline 1-oxide
}

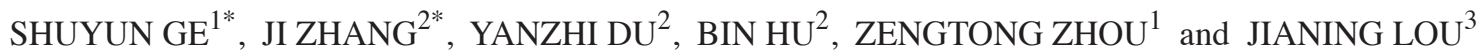 \\ ${ }^{1}$ Department of Oral Mucosal Diseases, Shanghai Key Laboratory of Stomatology, \\ Shanghai Ninth People's Hospital, Shanghai Jiao Tong University School of Medicine, Shanghai 200011; \\ ${ }_{2}^{2}$ State Key Laboratory of Medical Genomics, Ruijin Hospital, Shanghai Jiao Tong University School of Medicine, \\ Shanghai 200025; ${ }^{3}$ Department of Stomatology, Shanghai First People's Hospital, \\ Shanghai Jiao Tong University, Shanghai 200080, P.R. China
}

Received May 12,2015; Accepted January 7, 2016

DOI: $10.3892 / \mathrm{mmr} .2016 .4883$

\begin{abstract}
The typical progression of oral cancer is from hyperplastic epithelial lesions through dysplasia to invasive carcinoma. It is important to investigate malignant oral cancer progression and development in order to determine useful approaches of prevention of dysplastic lesions. The present study aimed to gain insights into the underlying molecular mechanism of oral carcinogenesis by establishing a rat model of oral carcinogenesis using 4-nitroquinoline 1-oxide. Subsequently, transcription profile analysis using an integrating microarray was performed. The dynamic gene expression changes of the six stages of rat oral carcinogenesis (normal, mild epithelial dysplasia, moderate dysplasia, severe dysplasia, carcinoma in situ and oral squamous cell carcinomas) were analyzed using component plane presentations (CPP)-self-organizing map (SOM). Six genes were verified by quantitative polymerase chain reaction, immunohistochemistry and succinate dehydrogenase (SDH) activity assay kit. Numerous differentially expressed genes (DEGs) were identified during rat oral carcinogenesis. CPP-SOM determined that these DEGs were primarily enriched during cell cycle, apoptosis, inflammatory response and tricarboxylic acid cycle, indicating the coordinated regulation of molecular networks. In addition, the expression of specific DEGs, such as janus kinase 3, cyclin-dependent kinase A-1, B-cell chronic lymphocytic leukaemia/lymphoma 2-like 2, nuclear factor- $\kappa \mathrm{B}$, tumor necrosis factor receptor superfamily member $1 \mathrm{~A}$, cyclin D1 and SDH were identified to have high concordance
\end{abstract}

Correspondence to: Dr Jianing Lou, Department of Stomatology, Shanghai First People's Hospital, Shanghai Jiao Tong University, 100 Haining Road, Shanghai 200080, P.R. China

E-mail: loujnjt@163.com

${ }^{*}$ Contributed equally

Key words: tricarboxylic acid cycle, microarray, 4-nitroquinoline 1-oxide, oral carcinogenesis with the results from microarray data. The current study demonstrated that oral carcinogenesis is a multi-step and multi-gene process, with a distinct pattern alteration along a continuum of malignant transformation. In addition, this comprehensive investigation provided a theoretical basis for the understanding of the molecular alterations associated with oral carcinogenesis.

\section{Introduction}

Oral cancer is the sixth most common type of cancer worldwide with annual incidence of $\sim 275,000$, and three quarters of all cases occur in developing countries (1). Oral cancer accounts for $2 \%$ of systemic malignant tumors; $90 \%$ of such patients are diagnosed with squamous cell carcinomas (SCC) (2). Although treatment strategies have progressed during the last 40 years, including surgery, radiation and chemotherapy, the five-year survival rate and life quality of patients with oral cancer remains unsatisfactory (2). In addition, these treatments often result in loss of speech, chewing and swallowing dysfunction, cosmetic deformity, and psychological distress (3). However, the effective management of patients with oral SCC is limited to the current knowledge of malignant tumor progression. Therefore, it is important to develop useful approaches to prevent dysplastic lesions, improve the accuracy of diagnosis, and determine definitive biological markers for the progression of these lesions to carcinomas.

The etiology of oral cancer is complex and is mediated by genetic-environmental interactions $(4,5)$. Numerous risk factors have been demonstrated to be associated with oral cancer, including tobacco and alcohol, dietary deficiencies, syphilis, human papillomavirus and chronic candidiasis (4). Previous studies have focused on genetic susceptibility and alterations, genomic instability, and epigenetic modifications in oral oncogenesis (6-8). Certain aberrantly expressed genes and proteins have been identified during oral cancer development, such as transforming growth factor- $\alpha$ (9), epidermal growth factor receptor (EGFR) (10), Ras (11), cadherin 1 type I (12), Bcl2-associated X protein and B-cell chronic lymphocytic leukaemia/lymphoma 2 (13). Although these studies contribute to the current understanding of the disease, 
the complexities of such malignancies are still not thoroughly elucidated. Genechip, or DNA microarray, allows for the simultaneous determination of the expression of tens of thousands of genes (14). This has revolutionized the screening for oral carcinogenesis-associated genes, in addition to accelerating the identification of potential therapeutic target genes (15). Hwang et al (16) used microarrays to evaluate overexpressed genes in oral cancer, and identified 45 genes, including two uncharacterized clones, that are associated with malignancy. Alevizos et al (17) determined that there are 600 differentially expressed genes (DEGs), including transcription factors, oncogenes, differentiation markers, tumor suppressors and metastatic proteins, in oral cancer. However, few studies have investigated the dynamic changes of gene expression during oral carcinogenesis.

In the present study, 4-nitroquinoline 1-oxide (4-NQO) was used to induce rat oral carcinogenesis. This animal model was selected due to its reproducibility and the anatomical similarities to humans (18), as well as the fact that it is widely used for investigations of oral cancer development. Subsequently, the dynamic changes of the gene expression profiles during the initiation and progression of oral cancer in Wistar rats were evaluated using microarray analysis. The current study aimed to define the genetic portrait of the different stages in oral SCC and identify oral carcinogenesis-associated genes for future studies, with the intent of exploring their potential roles during the progression of oral carcinogenesis and as possible target genes for the prevention of this disease.

\section{Materials and methods}

Animals and experimental design. A total of 38 healthy Wistar rats (160 days old, $220 \pm 10 \mathrm{~g}$ ) derived from closed groups were enrolled in the present study. The rats were acclimatized under appropriate conditions with a natural day-night cycle, with free access to food and water, at a temperature of $23 \pm 2^{\circ} \mathrm{C}$ and $30-50 \%$ humidity for 1 week prior to the trial. All animals and experimental procedures were approved by the Management Committee of Laboratory Animals Use, Institute of Laboratory Animals, Shanghai JiaoTong University (Shanghai, China).

4-NQO (Sigma-Aldrich, St. Louis, MO, USA) was dissolved in distilled water at a concentration of $0.002 \%$ and then stored in brown bottle at $4^{\circ} \mathrm{C}$. A total of 38 rats were randomly divided into the following two groups: i) The control group $(n=5)$, in which rats were treated with saline solution by drinking water; and ii) in the experimental group $(n=33)$, in which rats were treated with 4-NQO solution in the same way. Next, the rats in the 4-NQO group were randomly sacrificed by cervical dislocation at $9(n=7), 13(n=7), 20(n=5), 24(n=6)$ and $32(n=8)$ weeks, respectively. Tongue tissue from the most notable lesion site was collected and separated into the following three groups where the tissues were: i) Fixed with $10 \%$ buffered formalin (Sigma-Aldrich) for histopathological analysis; ii) immediately immersed in RNAlater solution (Qiagen $\mathrm{GmbH}$, Hilden, Germany) to ensure the stability of RNA, and frozen at $-80^{\circ} \mathrm{C}$; or iii) used to detect the activity of succinate dehydrogenase (SDH).

Pathological examination. The histological identification of squamous neoplasia was performed by a pathologist who was independent and blind to the study design. The samples were fixed in $10 \%$ buffered formalin, embedded with paraffin and then sliced into $5-\mu \mathrm{m}$ thick sections using a paraffin slicing machine (Leica Microsystems, Wetzlar, Germany). Next, sections were incubated for $4 \mathrm{~h}$ for deparaffinization at $65^{\circ} \mathrm{C}$ then were dehydrated with gradient ethanol. Subsequently, the sections were stained with hematoxylin (Genmed Scientifics, Inc., Shanghai, China) for $5 \mathrm{~min}$. Following differentiation in $1 \%$ hydrochloric acid alcohol for $2 \mathrm{sec}$, the sections were incubated in ammonia water for $2 \mathrm{~min}$ and stained with eosin (Genmed Scientifics, Inc.) for $1 \mathrm{~min}$. The sections were then dehydrated, cleared and mounted with neutral resin (Genmed Scientifics, Inc.). Light microscopy (BX50; Olympus, Tokyo, Japan) was used to observe the sections and the samples were classified into the following five types: i) Mild epithelial dysplasia (MiD), ii) moderate epithelial dysplasia (MoD), iii) severe epithelial dysplasia (SD), iv) carcinoma in situ (CIS); and v) SCC, according to the criteria described by the World Health Organization (19).

Microarrays and target sample preparation. Transcription profile analysis was performed using a Codelink Uniset Rat I Bioarray (GE Healthcare Life Sciences, Chandler, AZ, USA) containing 5,800 probes. Under RNase-free conditions, the samples were immersed into TRIzol solution (Invitrogen; Thermo Fisher Scientific, Inc., Waltham, MA, USA) and then homogenized on ice in a Dounce tissue grinder (Wheaton, Millville, NJ, USA). Total RNA was extracted according to the TRIzol extraction protocol, and then purified using an RNeasy kit (Qiagen $\mathrm{GmbH}$ ). For the microarrays, $10 \mu \mathrm{g}$ total RNA was used to synthesize cRNA by Codelink Expression Assay Reagent kit (GE Healthcare Life Sciences), according to the manufacturer's protocol. Briefly, single-stranded cDNA was generated using T7 primer and M-MLV reverse transcriptase (Promega Corp., Madison, WI, USA), and then double-stranded cDNA was produced using RNase $\mathrm{H}$ and DNA polymerase I (Invitrogen; Thermo Fisher Scientific, Inc.). Subsequently, cRNA was generated based on the double-stranded cDNA template using biotin-11-uridine-5'-triphosphate and T7 RNA polymerase in an in vitro transcription reaction. The purification and quantitation of cRNA was performed using an RNeasy kit (Qiagen GmbH) and UV spectrophotometry (Evolution 300; Thermo Fisher Scientific, Inc.), respectively.

Hybridization, processing and scanning. A total of $10 \mu \mathrm{g}$ cRNA was fragmented by incubation with $50 \mu$ l fragmentation buffer (Codelink Expression Assay Reagent kit) for $20 \mathrm{~min}$ at $94^{\circ} \mathrm{C}$. The fragmented cRNA in hybridization buffer was hybridized to the Uniset bioarray in a shaking incubator (overnight, $300 \mathrm{rpm}$ at $37^{\circ} \mathrm{C}$ ). Arrays were washed with phosphate-buffered saline (PBS) three times, and then stained with Cy5-Streptavidin (Invitrogen; Thermo Fisher Scientific, Inc.) for $30 \mathrm{~min}$. Subsequently, arrays were washed and then dried by centrifugation at $1,000 \mathrm{x}$ g for $3 \mathrm{~min}$ at low speed. The arrays were scanned using a GenePix 4000B microarray scanner (Molecular Devices, LLC, Sunnyvale, CA, USA).

Data analysis. Data preprocessing was performed using CodeLink Expression Analysis software (version 2.2.3; GE Healthcare Life Sciences) (20). Probe-level data were 
extracted and then normalized using linear median normalization. Following the removal of low hybridization signals, normalized data were converted into approximately normal distribution from skewed distribution using a $\log _{2}$ transformation. DEGs were selected on the basis of a fold-change $>1.5$ and $\mathrm{P}<0.05$. The software package of self-organizing map (SOM; http://www.cis.hut.fi/projects/somtoolbox/) based on the Matlab (version 6.5; MathWorks, Natick, MA, USA) environment was used for data mining and gene clustering, in accordance with a previous study (21). Data were processed using 400 (24x15 grids) neurons. The SOM illustration output was visualized using component plane presentations (CPP) based on a Matlab 6.5 environment (21). To characterize gene expression patterns, the clusters were annotated with Gene Ontology (GO; http://geneontology.org/) enrichment analysis based on Database for Annotation, Visualization and Integrated Discovery (22).

Quantitative polymerase chain reaction $(q P C R)$. The six randomly selected DEGs were Janus kinase 3 (Jak3), cyclin-dependent kinase-1 (CDK1; also known as Cdc2a), Bcl2-like 2 ( Bcl212), nuclear factor- $\kappa \mathrm{B}(\mathrm{NF}-\kappa \mathrm{B})$, tumor necrosis factor receptor superfamily, member 1A (Tnfrsfla), and SDH were detected by qPCR. PCR amplification was performed using an iCycler iQ Real-Time PCR detection system (Bio-Rad Laboratories, Richmond, CA, USA) with SYBR-Green I fluorescent dye (Applied Biosystems; Thermo Fisher Scientific, Inc.). Primer sequences for Jak3, Cdc2a, Bcl212, NF- $\kappa$ B, Tnfrsfla, SDH and 18S RNA are presented in Table I. The PCR program included $95^{\circ} \mathrm{C}$ for $10 \mathrm{~min}$, 40 cycles at $95^{\circ} \mathrm{C}$ for $15 \mathrm{sec}, 55^{\circ} \mathrm{C}$ for $1 \mathrm{~min}$, and a final $20 \mathrm{~min}$ extension at $60-95^{\circ} \mathrm{C}$. Finally, the expression of each gene was calculated using the $2^{-\Delta \Delta \mathrm{Cq}}$ method (23) and $18 \mathrm{~S}$ RNA was used as the internal control.

Immunohistochemical (IHC) staining. The 5- $\mu \mathrm{m}$ sections were incubated with $0.3 \%$ hydrogen peroxide in PBS for 5 min to block the endogenous peroxidase. An antigen retrieval step was performed in a microwave $(700 \mathrm{~W})$ with $10 \mathrm{mM}$ citrate buffer ( $\mathrm{pH}$ 6.0; GenMed Scientifics, Inc.) for 5-10 min. The sections were blocked using 5\% normal goat serum (GenMed Scientifics, Inc.) in PBS for $10 \mathrm{~min}$, and then incubated with mouse anti-rat cyclin D1 monoclonal antibody (1:50; cat. no. sc-20044; Santa Cruz Biotechnology, Inc., Dallas, TX, USA) and polyclonal rabbit anti-rat NF- $\kappa$ B 65 antibody (1:50; cat. no. sc-372; Santa Cruz Biotechnology, Inc.) for $2 \mathrm{~h}$ at room temperature. Subsequently, the sections were incubated with biotin-labeled goat anti-mouse IgG1-B (1:200; cat. no. sc-2072; Santa Cruz Biotechnology, Inc.) and mouse anti-rabbit IgG-B (1:200; cat. no. sc-2491; Santa Cruz Biotechnology, Inc.) polyclonal secondary antibodies for $15 \mathrm{~min}$, respectively, and then incubated with streptavidin for 15 min (SP kit; Zymed; Thermo Fisher Scientific, Inc.) at room temperature. In addition, an antigen retrieval step was performed in a microwave $(700 \mathrm{~W})$ with $10 \mathrm{mM}$ citrate buffer (pH 6.0; GenMed Scientifics) for 5-10 min, which aimed to expose antigen sites masked by fixation. Immunolabeling was visualized by incubating the tissue sections with 3,3'-diaminobenzidine solution for $15 \mathrm{~min}$ at room temperature and counterstaining with hematoxylin for $2 \mathrm{~min}$ at room
Table I. Primer sequences for specific genes.

\begin{tabular}{ll}
\hline Gene & \multicolumn{1}{c}{ Primer sequences $\left(5^{\prime}-3^{\prime}\right)$} \\
\hline Jak3 & F: CCTGGAGTGGCACGAGAATC \\
& R: TCCACAACCTCCCGCCTAT \\
Fdc2a & F: CCGGTTGACATCTGGAGCAT \\
& R: CTGAGTCGCCGTGGAAAAG \\
Bcl212 & F: TCCGAGTTCCGGGAAGACT \\
& R: TGGTAACCCGACCCTGGTT \\
NF-kB & F: CCAACGCCCTCTTCGACTAC \\
& R: CCTCACGAGCTGAGCATGAA \\
Tnfrsf1a & F: TCAATGGCACCGTGACAATC \\
& R: AAGAATCCTGCGTGGCAGTT \\
SDH & F: CATGGCGACTGCCTATGCT \\
& R: CCTTCGTTCTTCAGCCGCT \\
18 RNA & F: TGTCGCTCGCTCCTCTCCTA \\
& R: TGACCGGGTTGGTTTTGATC
\end{tabular}

Jak3, Janus kinase 3; Cdc2a, cyclin-dependent kinase-1; Bcl212, Bcl2-like 2; NF- $\mathrm{B}$, nuclear factor- $\mathrm{B}$; Tnfrsfla, tumor necrosis factor receptor superfamily, member $1 \mathrm{~A} ; \mathrm{SDH}$, succinate dehydrogenase.

temperature. The primary antibody was replaced with $0.01 \mathrm{M}$ PBS in the negative controls. Light microscopy (BX50; Olympus) was used to observe the sections.

Detection of SDH activity. Tongue tissues were homogenized on ice in a Dounce tissue grinder, and then tissue mitochondria were separated and extracted using tissue mitochondria isolation kit (GenMed Scientifics), according to manufacturer's protocol. Subsequently, the activity of mitochondrial SDH was detected six times using SDH activity assay kit (GenMed Scientifics), according to the manufacturer's protocol. The absorbance was read at $600 \mathrm{~nm}$ using a microplate reader (SpectraMax 190; Molecular Devices, LLC).

Statistical analysis. Statistical analysis was performed using SPSS software (version 17.0; SPSS, Inc., Chicago, IL, USA). Data are presented as the mean \pm standard deviation and were analyzed by independent sample t-tests. $\mathrm{P}<0.05$ was considered to indicate a statistically significant difference.

\section{Results}

Histological alterations to the mucosa following $4 N Q O$-administration. Following treatment of rat tongue tissues with 4-NQO for 9 weeks, hematoxylin and eosin staining demonstrated a number of initial alterations, including an increased thickness of the spinous cell layer and disordered basal cells; of these tissues, were diagnosed as MiD. Following treatment with $4 \mathrm{NQO}$ for 13 weeks, further dysplastic alterations occurred, and MoD, SD, CIS and invasive SCC were additionally observed in the majority of rats tongue tissues sampled following treatment for 20, 24 and 32 weeks (Fig. 1). The histological types in each group are summarized in Table II. 
Data mining and visualization by CPP-SOM. A total of 492, 497, 1,176, 1,479 and 1,848 DEGs were identified in the tongue tissues of MiD, MoD, SD, CIS and SCC-stage rats. There were a similar number of up- and downregulated genes in each of the tongue tissues types (Fig. 2A). Gene clustering and its visualization are indicated in Fig. 2B using CPP-SOM. Each bar in the graphic illustrates the values of a single vector component in all map units. DEGs mapped at each stage during rat oral carcinogenesis indicated that the genes mapped onto the corner/edge areas of the map were most frequently regulated (Fig. 2B).

GO enrichment analysis of DEGs in tongue tissues at each stage during rat tongue carcinogenesis. As presented in Fig. 3A, downregulated genes at the MiD and MoD stage (cluster 1) were involved in electron transport and mitochondria respiration complexes, while upregulated genes (cluster 7) were involved in the immune and inflammatory responses, such as interleukin-1 (IL-1), CD40 and chemokine (C-X-C motif) ligand 10. Down/upregulated genes at the SCC stage (cluster 2/6) were associated with carboxylic acid, amino acid and amine metabolism and the apoptosis pathway, respectively. Upregulated genes at the SD and CIS stage (cluster 4) were associated with cell-cell signaling and small GTPase mediated signal transduction. In addition, down/upregulated genes at the SD and CIS stage (cluster 3/5) were enriched in cellular metabolism and transporter activity, as well as cell cycle and DNA repair. Dynamic gene alterations underlying tricarboxylic acid (TCA) cycle (Fig. 3B) and DNA repair (Fig. 3C) were also highlighted during rat oral carcinogenesis.

Verification of five randomly selected DEGs expression levels by $q P C R$. According to $\mathrm{qPCR}$ analysis, the mRNA levels of Jak3, Bcl2I2 and NF- $\mathrm{B}$ were significantly higher with the development of rat oral carcinogenesis $(\mathrm{P}<0.05)$, while mRNA levels of Cdc2a and Tnfrsfla were significantly elevated at the early and intermediate stages but were reduced at the late stage $(\mathrm{P}<0.01 ; \mathrm{P}<0.05)$ during rat oral carcinogenesis (Fig. 4). These results are in high concordance with the results from microarray analysis.

Expression of $N F-\kappa B p 65$ and cyclin D1 in tongue tissues at each stage during rat tongue carcinogenesis. IHC with anti-NF- $\mathrm{Bp} 65$ antibody indicated the presence of $\mathrm{NF}-\kappa \mathrm{Bp} 65$-labeled cells were identified a fine granular pattern. The normal sample indicated no expression of $\mathrm{NF}-\kappa \mathrm{Bp} 65$ in the granular layer. However, in the mild and moderate dysplasia epithelium, the expression of NF- $\mathrm{NBp} 65$ was observed in the basal cell layer (Fig. 5A). In addition, the expression of NF- $\mathrm{NBp} 65$ marginally increased in parallel with the severity of dysplasia. Compared with SCC, most samples of dysplasia epithelium had a weaker expression of NF-кBp65 (Fig. 5B). This result was consistent with the results of the $\mathrm{qPCR}$.

In normal epithelium, the expression of cyclin D1 was only observed in the nuclei of basal cell layer. In dysplastic epithelium, the levels of the cyclin D1 protein markedly increased, and the distribution of positive cells extended from the basal cell layer to the lower spinous cell layer (Fig. 5C).
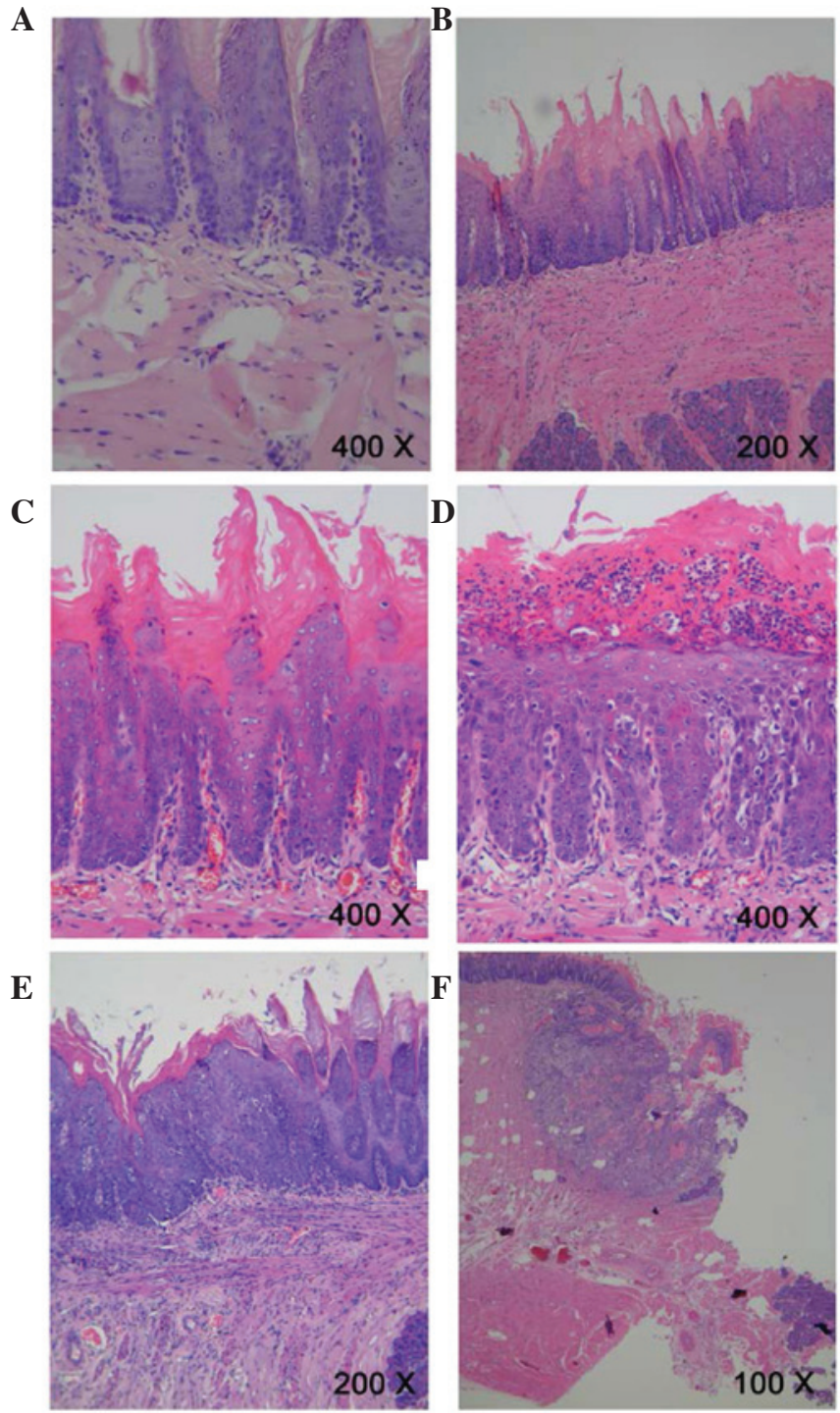

Figure 1. Pathological evidence of carcinogenesis in rat tongues. (A) Normal squamous epithelium of the tongue. Magnification, x400. (B) Mild epithelial dysplasia of the tongue exhibited loss of polarity of the deeper cell layers of the epithelium and mild nuclear pleomorphism of cells. Magnification, x200. (C) Moderate epithelial dysplasia of the tongue indicated a basaloid appearance, loss of polarity of cells and intercellular cohesion. Magnification, x400. (D) Severe epithelial dysplasia of the tongue exhibited a proliferation of basal cells, grossly disturbed stratification, the loss of polarity of cells, individual cell keratinization and nuclear pleomorphism of cells. Magnification, x400. (E) Carcinoma in situ of the tongue exhibited the proliferation of primitive basal epithelial cells from the basement membrane to the surface, marked nuclear atypia and the full thickness of the epithelium. Magnification, x200. (F) Squamous cell carcinoma of the tongue showed gross disruption of normal epithelial architecture, prominent cellular pleomorphism, the formation of dyskeratosis with keratin pearl and invasion into underlying connective tissues. Magnification, x100.

In the sample of SCC, cyclin D1-labeled cells were localized in the peripheral layer of tumor nests (Fig. 5D).

mRNA level of SDH and mitochondrial SDH activity in tongue tissues at each stage during rat tongue carcinogenesis. qPCR analysis indicated that the mRNA expression level of SDH was significantly reduced in tongue tissues during rat oral carcinogenesis $(\mathrm{P}<0.05$; Fig. 6A). Furthermore, compared with normal tissues, the activity of mitochondrial SDH was also reduced during carcinogenesis $(\mathrm{P}<0.05$; Fig. 6B). 
Table II. Histological changes in rat tongues at various time points following 4-nitroquinoline 1-oxide treatment.

\begin{tabular}{lccccccc}
\hline & \multicolumn{7}{c}{ Diagnosis } \\
\cline { 2 - 5 } Group (weeks) & Normal & Mild dysplasia & Moderate dysplasia & Severe dysplasia & Carcinoma in situ & SCC & Total \\
\hline $0($ Control) & $5(100)$ & $0(0)$ & $0(0)$ & $0(0)$ & $0(0)$ & $0(0)$ & 5 \\
9 & $0(0)$ & $4(57.1)$ & $3(42.9)$ & $0(0)$ & $0(0)$ & $0(0)$ & 7 \\
13 & $0(0)$ & $1(14.3)$ & $5(71.4)$ & $1(14.3)$ & $0(0)$ & $0(0)$ & 7 \\
20 & $0(0)$ & $0(0)$ & $0(0)$ & $5(100)$ & $0(0)$ & $0(0)$ & 5 \\
24 & $0(0)$ & $0(0)$ & $0(0)$ & $2(33.3)$ & $4(66.7)$ & $0(0)$ & 6 \\
32 & $0(0)$ & $0(0)$ & $0(0)$ & $0(0)$ & $2(25)$ & $6(75)$ & 8 \\
Total & 5 & 5 & 8 & 8 & 6 & 6 & 38 \\
\hline
\end{tabular}

Values are presented as $\mathrm{n}(\%$ of total of respective group). SCC, squamous cell carcinoma.

A

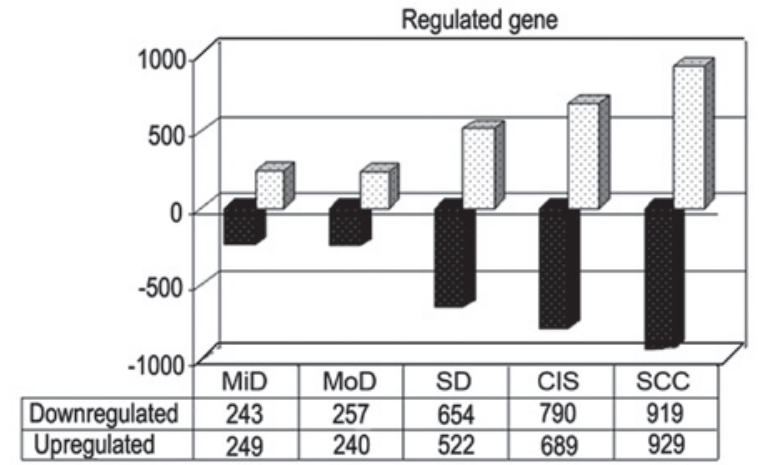

$\mathbf{B}$
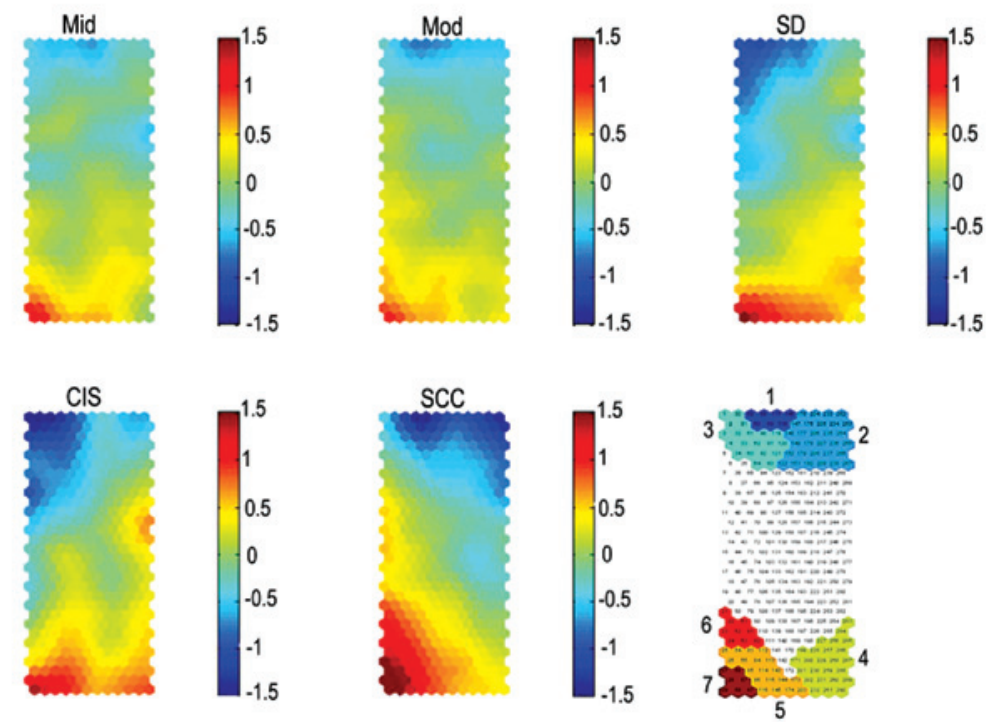

Figure 2. Differentially expressed genes and SOM output data at each stage during rat oral carcinogenesis. (A) The numbers of up/downregulated genes in tongue tissues at different stages during carcinogenesis. (B) Illustration of SOM output microarray data by component plane presentations. Each of these presentations appears as genome-wide transcriptional display, in which all upregulated units (red), downregulated units (blue) and moderately transcribed units (green and yellow) are well-delineated. Color-coding index represents $\log _{2}$-transformed ratios, and the brighter color denotes the higher value. The following 7 major regulatory categories are recognizable: i) 1/7, Down/upregulated genes at the early stage (MiD and MoD); ii) $2 / 6$, down/upregulated genes at the late stage (SCC); iii) 3/5, down/upregulated genes at the intermediate stage (SD and CIS); iv) 4, upregulated genes at the intermediate stage (SD and CIS). SOM, self-organizing map; MiD, mild epithelial dysplasia; MoD, moderate epithelial dysplasia; SCC, squamous cell carcinoma; SD, severe epithelial dysplasia; CIS, carcinoma in situ.

\section{Discussion}

In the current study, the administration of $4 \mathrm{NQO}$ produced a successive oral carcinogenesis progression model, including multiple dysplastic, preneoplastic and neoplastic lesions, following treatment for 9, 13, 20, 24 and 32 weeks. These sequential changes in the epithelial cells mimicked human oral cavity neoplastic transformation. Subsequently, 492, 497, 



C

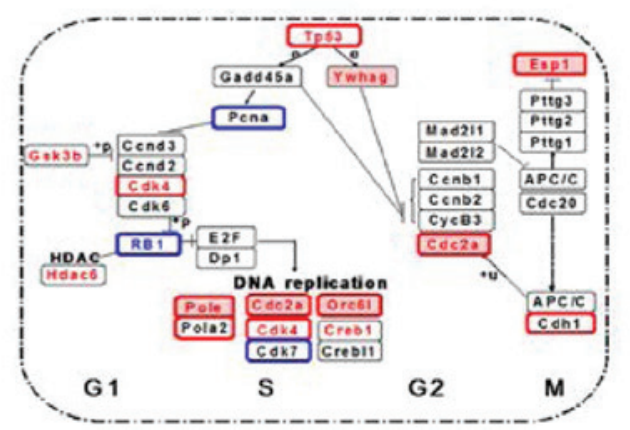

Figure 3. Functional enrichment of DEGs during rat oral carcinogenesis. (A) Gene ontology enrichment analysis of DEGs at different pathological stage. Illustration of DEGs underlying (B) TCA cycle and and (C) cell cycle during oral carcinogenesis. DEGs at the early stage are highlighted by background pink (upregulation) and light-blue (downregulation), whereas DEGs at intermediate and late stage are indicated by red (upregulation) and blue (downregulation). Synergistically/additively impacted genes in the cotreatment series are designated with asterisks. Unchanged/unmentioned genes in the experimental data are marked and framed by black with light background. DEG, differentially expressed gene; TCA, tricarboxylic acid cycle.

$1,176,1,479$ and 1,848 DEGs were identified in tongue tissues of MiD, MoD, SD, CIS and SCC, respectively. CPP-SOM gene expression data from the five stages of disease analyzed, indicated a distinct separation between normal, mild to moderate epithelial dysplasia and SD to SCC. Therefore, oral carcinogensis was divided into three stages at the genome-wide level: i) Initiation, early stage (MiD and MoD); ii) promotion, intermediate stage (SD and CIS); and iii) progression, late stage (SCC). The changes in the DEGs in these five stages also confirmed that MiD and MoD were not only an important early stage, but also an important turning point for oral carcinogenesis. Additionally, it was determined that these DEGs were primarily enriched during the cell cycle, apoptosis, inflammatory response and TCA cycle. The expression levels of certain DEGs, such as Jak3, Cdc2a, Bcl2I2, NF-кB, Tnfrsfla, CCND1 and SDH, indicated a high concordance with the results from microarray data.

Dysregulation of cell proliferation and apoptosis is considered as a primary event in cancer development (24). In the present study, transcriptional changes of the cell cycle and apoptosis-associated genes primarily occurred at the intermediate and later stage of disease onset. In normal cells, DNA replication is tightly monitored by cell-cycle checkpoints. Cyclin D1 (CCND1; also known as the Bcl-1 gene), was a positive regulator of $\mathrm{G}_{1}$ phase (25), and overexpressed cyclin D1 may increase cancer progression through promoting 


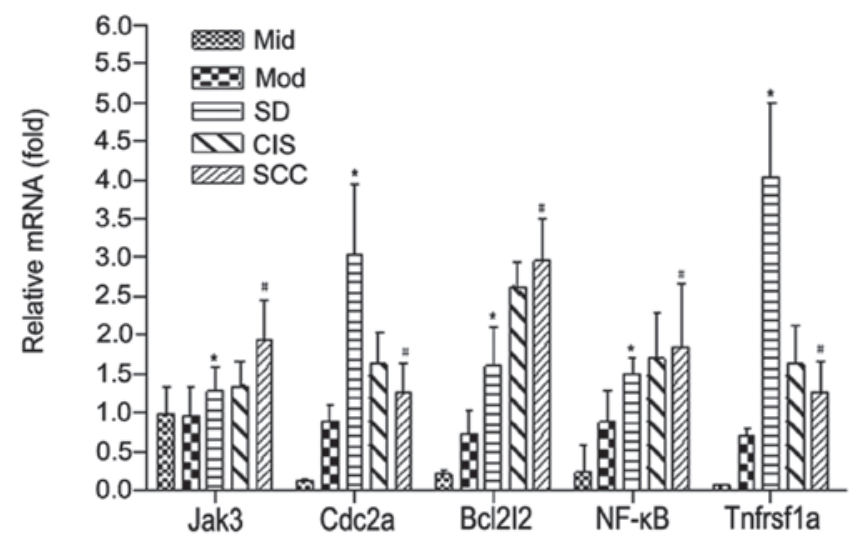

Figure 4. Verification of mRNA levels of Jak3, Cdc2a, Bcl2I2, NF- $\kappa \mathrm{B}$ and Tnfrsf1a by reverse transcription-quantitative polymerase chain reaction. ${ }^{*} \mathrm{P}<0.05$ vs. MiD and MoD, ${ }^{\#} \mathrm{P}<0.05$ vs. MiD, MoD and SD. Jak3, janus kinase 3; Cdc2a, cyclin dependant kinase; Bcl212, Bcl-2-like 2; NF- $\kappa$ B; nuclear factor- $\kappa$ B; Tnfrsf1a, tumor necrosis factor receptor 1; MiD, mild epithelial dysplasia; MoD, moderate epithelial dysplasia; SD, severe epithelial dysplasia; CIS, carcinoma in situ; SCC, squamous cell carcinoma.
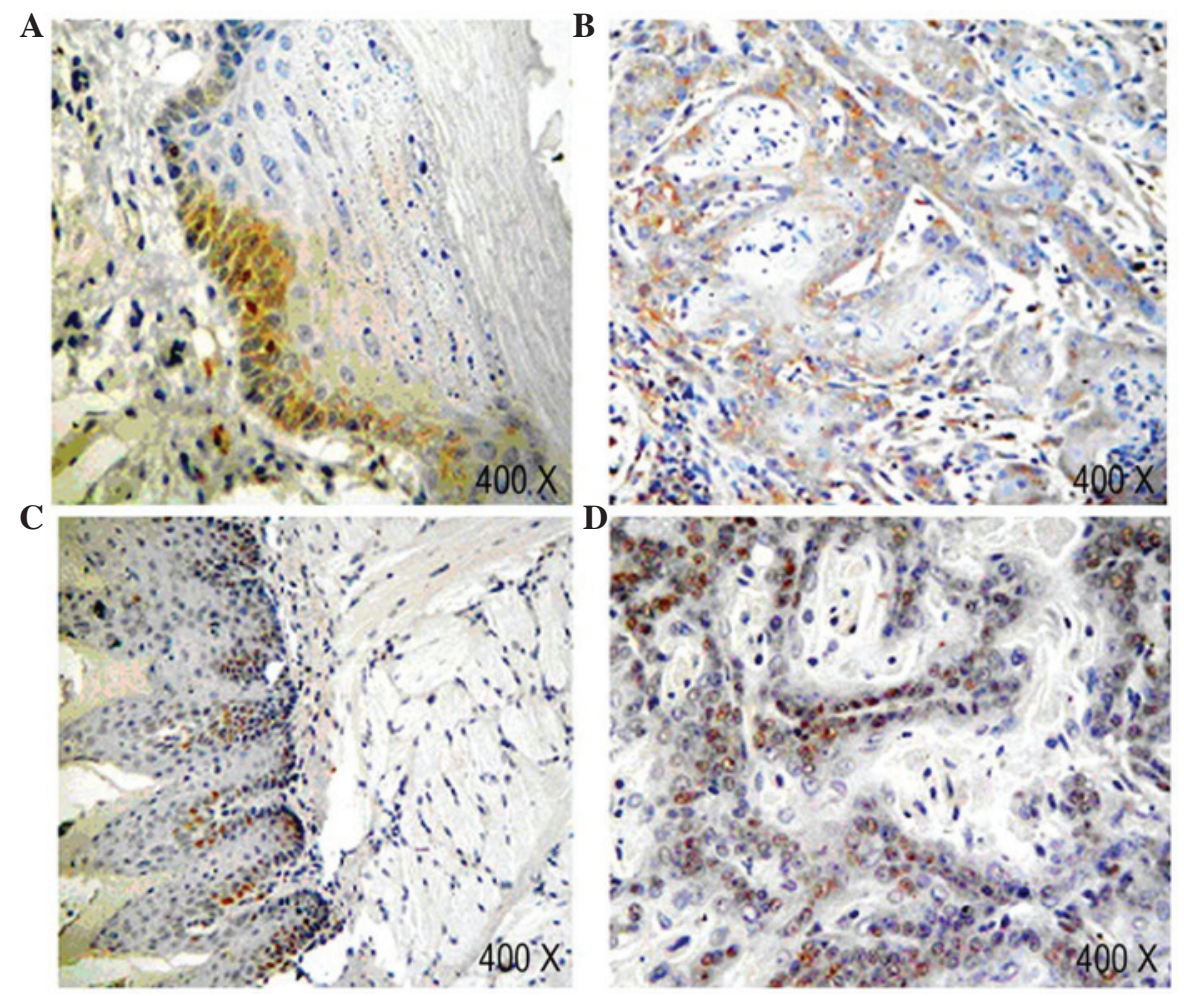

Figure 5. Immunohistochemical staining of NF- $\mathrm{kBp} 65$ and cyclin D1 in tongue tissues during rat oral carcinogenesis. (A) Immunostaining for NF- $\mathrm{kBp} 65$ in mild epithelial dysplasia tissue. (B) Immunostaining for NF-kBp65 in squamous cell carcinoma tissue. (C) Immunostaining for cyclin D1 in mild epithelial dysplasia tissue. (D) Immunostaining for cyclin D1 in squamous cell carcinoma tissue. Original magnification, x400. NF- $\kappa$ Bp65, nuclear factor- $\kappa B p 65$.
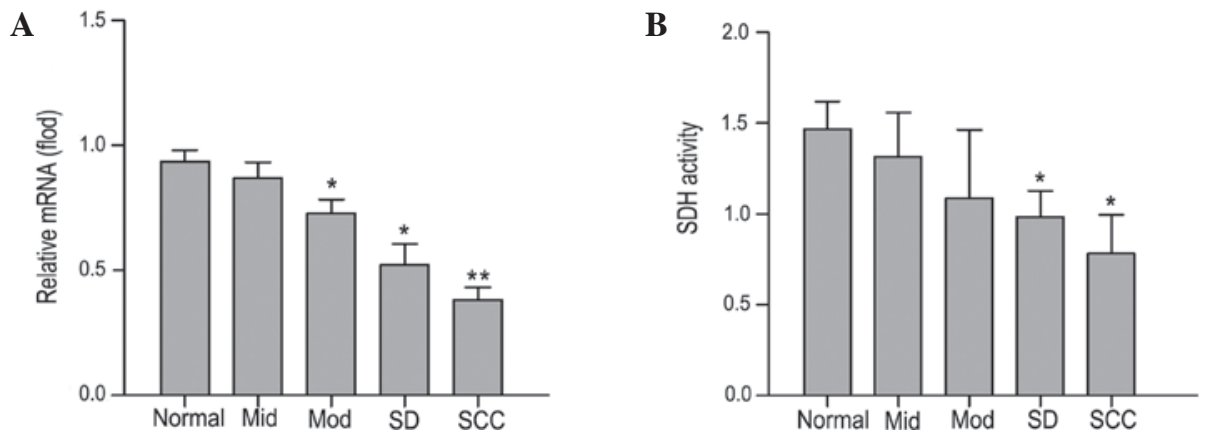

Figure 6.(A) mRNA expression and activity of (B) SDH during rat oral carcinogenesis. $\mathrm{P}<0.05$ and ${ }^{* *} \mathrm{P}<0.05$ vs. normal group. SDH, succinate dehydrogenase; Mid, mild epithelial dysplasia; Mod, moderate epithelial dysplasia; SD, severe epithelial dysplasia; SCC, squamous cell carcinoma. 
the $G_{1}$ phase of the cell cycle (26). In accordance with the results of the current study, the expression of CCND1 was increased in previous studies investigating oral cancer (27) and premalignant lesions (28). In addition, the Rb protein, as a key component of $\mathrm{G}_{1}$ checkpoints, may be activated to lead to $\mathrm{G}_{1}$ arrest by dephosphorylation (29). In addition, the present study demonstrated that important positive regulators of the cell cycle, including key regulators of $\mathrm{G}_{1} / \mathrm{S}$ transition (CDK2 and $\mathrm{CDK} 4$ ) and the $\mathrm{G}_{2} / \mathrm{M}$ transition (CDC2 and CCND2), were upregulated. The upregulation of such a large number of positive cell cycle regulators and the downregulation of negative regulators had a synergistic effect on cell proliferation. In addition, the present study also indicated that there were consequences of transcriptional remodeling of genes involved in apoptosis. Bcl-2, as an anti-apoptotic protein (30), was upregulated in the current study. All these results suggest that the cell cycle regulators and $\mathrm{Bcl}-2$ may accelerate cancer cell proliferation during oral carcinogenesis.

A previous study demonstrated that $\mathrm{NF}-\kappa \mathrm{B}$ is involved in immune and inflammatory reactions and cell differentiation, in addition to anti-apoptosis, by inducing the expression of various genes, such as tumor necrosis factor- $\alpha$ (TNF- $\alpha$ ), and IL-1, TNF receptor-associated factors (31). Constitutive NF- $\kappa \mathrm{B}$ activation had been identified in a large variety of human malignancies, such as acute lymphoblastic leukemia, breast cancer, colon cancer, liver cancer and melanoma (32-35). The present study determined that the mRNA and protein expression levels of $\mathrm{NF}-\kappa \mathrm{B}$ increased with the progression of oral carcinogenesis, which was consistent with the result of Nakayama et al (36). Additionally, it was determined that the mRNA levels of Tnfrsfla were also increased. Over the past decade, it has been demonstrated that TNF- $\alpha$ is an inflammatory cytokine important for the tumor initiation process (37). Consistent with the results of the current study, the expression of TNF- $\alpha$ was elevated in the saliva of patients with oral SCC compared with patients without oral lesions (38). These results indicated that $\mathrm{NF}-\kappa \mathrm{B}$ promoted an inflammatory response by regulating the expression of TNF- $\alpha$ during oral carcinogenesis.

In addition to cell proliferation, apoptosis and inflammatory reactions, the TCA cycle was also observed to be involved in the early stages of oral carcinogenesis. In the present study, the activity of key enzymes, such as SDH, isocitrate dehydrogenase, fumarate hydratase, malate dehydrogenase and pyruvate dehydrogenase were reduced during oral carcinogenesis. This may explain why cancer cell morphology and structure change and the number of mitochondria are markedly reduced in cancer cells.

SDH consists of four subunits (A, B, C and D), and acts as a mitochondrial TCA cycle enzyme and a tumor suppressor $(39,40)$. Inherited or somatic mutations in subunits B, C or D of SDH may lead to pheochromocytoma (41), paraganglioma (42) or renal cell carcinoma (43). The downregulation of SDH was observed in gastric carcinoma (44). Selak et al (40) determined that succinate, which accumulates as a result of SDH inhibition, inhibited hypoxia-inducible factor- $\alpha$ (HIF- $\alpha$ ) prolyl hydroxylase, and then led to the stabilization and activation of HIF- $\alpha$. Subsequently, HIF- $\alpha$ entered the nucleus and increased the expression of genes, such as platelet-derived growth-factor receptor and EGFR, which in turn facilitated glycolysis, metastasis and angiogenesis, eventually leading to tumor progression (40). In addition, as SDH is embedded in the inner mitochondrial membrane, mutant SDH subunits may alter the membrane composition, and thus lead to a mitochondrion resistant to apoptosis (45). This indicates that the TCA cycle may be closely associated with oral carcinogenesis through the regulation of SDH.

In summary, oral carcinogenesis is a multi-step and multi-gene process, with a distinct pattern of alteration along a continuum of malignant transformation. Further investigations are necessary to validate these observations, which may enhance the understanding of the molecular alterations associated with oral carcinogenesis.

\section{Acknowledgements}

The authors would like to thank Shanghai Biochip Co., Ltd. for their technical support. The present study was supported by grants from the Youth Project of the National Natural Science Foundation of China (grant no. 30700944) and the Shanghai Municipal Health Bureau (grant no. 2012092).

\section{References}

1. Ferlay J, Bray F, Parkin DM, Pisani P (eds): Globocan 2000: Cancer Incidence and Mortality Worldwide (IARC Cancer Bases No. 5). IARCPress, Lyon, 2001.

2. Warnakulasuriya S: Global epidemiology of oral and oropharyngeal cancer. Oral Oncol 45: 309-316, 2009.

3. Kademani D, Bell RB, Schmidt BL, Blanchaert R, Fernandes R, Lambert P and Tucker WM; American Association of Oral and Maxillofacial Surgeons Task Force on Oral Cancer: A preliminary report from the American Association of Oral and Maxillofacial Surgeons Task Force on Oral Cancer. J Oral Maxillofac Surg 66: 2151-2157, 2008.

4. Ram H, Sarkar J, Kumar H, Konwar R, Bhatt ML and Mohammad S: Oral cancer: Risk factors and molecular pathogenesis. J Maxillofac Oral Surg 10: 132-137, 2011.

5. Williams H: Molecular pathogenesis of oral squamous carcinoma. Mol Pathol 53: 165-172, 2000.

6. Nagpal JK and Das BR: Oral cancer: Reviewing the present understanding of its molecular mechanism and exploring the future directions for its effective management. Oral Oncol 39: 213-221, 2003.

7. Shaw R: The epigenetics of oral cancer. Int J Oral Maxillofac Surg 35: 101-108, 2006.

8. Scully C, Field JK and Tanzawa H: Genetic aberrations in oral or head and neck squamous cell carcinoma (SCCHN): 1. Carcinogen metabolism, DNA repair and cell cycle control. Oral Oncol 36: 256-263, 2000.

9. Todd R, Chou MY, Matossian K, Gallagher GT, Donoff RB and Wong DT: Cellular sources of transforming growth factor-alpha in human oral cancer. J Dent Res 70: 917-923, 1991.

10. Partridge M, Gullick WJ, Langdon JD and Sherriff M: Expression of epidermal growth factor receptor on oral squamous cell carcinoma. Br J Oral Maxillofac Surg 26: 381-389, 1988.

11. McDonald JS, Jones H, Pavelic ZP, Pavelic LJ, Stambrook PJ and Gluckman JL: Immunohistochemical detection of the H-ras, K-ras, and N-ras oncogenes in squamous cell carcinoma of the head and neck. J Oral Pathol Med 23: 342-346, 1994.

12. Williams HK, Sanders DS, Jankowski JA, Landini G and Brown AM: Expression of cadherins and catenins in oral epithelial dysplasia and squamous cell carcinoma. J Oral Pathol Med 27: 308-317, 1998.

13. Ravi D, Ramadas K, Mathew BS, Nalinakumari KR, Nair MK and Pillai MR: De novo programmed cell death in oral cancer. Histopathology 34: 241-249, 1999.

14. Duggan DJ, Bittner M, Chen Y, Meltzer P and Trent JM: Expression profiling using cDNA microarrays. Nat Genet 21 (Suppl): 10-14, 1999.

15. Kuo WP, Whipple ME, Sonis ST, Ohno-Machado L and Jenssen TK: Gene expression profiling by DNA microarrays and its application to dental research. Oral Oncol 38: 650-656, 2002. 
16. Hwang D, Alevizos I, Schmitt WA, Misra J, Ohyama H, Todd R Mahadevappa M, Warrington JA, Stephanopoulos G, Wong DT and Stephanopolus G: Genomic dissection for characterization of cancerous oral epithelium tissues using transcription profiling. Oral Oncol 39: 259-268, 2003.

17. Alevizos I, Mahadevappa M, Zhang X, Ohyama H, Kohno Y, Posner M, Gallagher GT, Varvares M, Cohen D, Kim D, et al: Oral cancer in vivo gene expression profiling assisted by laser capture microdissection and microarray analysis. Oncogene 20: 6196-6204, 2001.

18. Tang XH, Knudsen B, Bemis D, Tickoo S and Gudas LJ: Oral cavity and esophageal carcinogenesis modeled in carcinogen-treated mice. Clin Cancer Res 10: 301-313, 2004

19. Kramer IR, Lucas RB, Pindborg JJ and Sobin LH: Definition of leukoplakia and related lesions: An aid to studies on oral precancer. Oral Surg Oral Med Oral Pathol 46: 518-539, 1978.

20. Horcajadas JA, Sharkey AM, Catalano RD, Sherwin JR Domínguez F, Burgos LA, Castro A, Peraza MR, Pellicer A and Simón C: Effect of an intrauterine device on the gene expression profile of the endometrium. J Clin Endocrinol Metab 91: 3199-3207, 2006.

21. Xiao L, Wang K, Teng Y and Zhang J: Component plane presentation integrated self-organizing map for microarray data analysis. FEBS Lett 538: 117-124, 2003.

22. Dennis G Jr, Sherman BT, Hosack DA, Yang J, Gao W, Lane HC and Lempicki RA: DAVID: Database for annotation, visualization, and integrated discovery. Genome Biol 4: 3, 2003.

23. Livak KJ and Schmittgen TD: Analysis of relative gene expression data using real-time quantitative PCR and the $2-\Delta \Delta \mathrm{Ct}$ method. Methods 25: 402-408, 2001.

24. Evan GI and Vousden KH: Proliferation, cell cycle and apoptosis in cancer. Nature 411: 342-348, 2001.

25. Smith BD and Haffty BG: Molecular markers as prognostic factors for local recurrence and radioresistance in head and neck squamous cell carcinoma. Radiat Oncol Investig 7: 125-144, 1999.

26. Quelle DE, Ashmun RA, Shurtleff SA, Kato JY, Bar-Sagi D, Roussel MF and Sherr CJ: Overexpression of mouse D-type cyclins accelerates G1 phase in rodent fibroblasts. Genes Dev 7: $1559-1571,1993$.

27. Miyamoto R, Uzawa N, Nagaoka S, Hirata Y and Amagasa T: Prognostic significance of cyclin D1 amplification and overexpression in oral squamous cell carcinomas. Oral Oncol 39: 610-618, 2003

28. Robinson CM, Prime SS, Huntley S, Stone AM, Davies M, Eveson JW and Paterson IC: Overexpression of JunB in undifferentiated malignant rat oral keratinocytes enhances the malignant phenotype in vitro without altering cellular differentiation. Int J Cancer 91: 625-630, 2001.

29. Cheng YH, Li LA, Lin P, Cheng LC, Hung CH, Chang NW and Lin C: Baicalein induces G1 arrest in oral cancer cells by enhancing the degradation of cyclin D1 and activating AhR to decrease $\mathrm{Rb}$ phosphorylation. Toxicol Appl Pharmacol 263: 360-367, 2012

30. Wilson GD, Saunders MI, Dische S, Richman PI, Daley FM and Bentzen SM: bcl-2 expression in head and neck cancer: An enigmatic prognostic marker. Int J Radial Oncol Biol Phys 49: 435-441, 2001
31. May MJ and Ghosh S: Signal transduction through NF-kappaB. Immunol Today 19: 80-88, 1998.

32. Tracey L, Streck CJ, Du Z, Williams RF, Pfeffer LM, Nathwani AC and Davidoff AM: NF-kappaB activation mediates resistance to IFNbeta in MLL-rearranged acute lymphoblastic leukemia. Leukemia 24: 806-812, 2010.

33. Shostak $\mathrm{K}$ and Chariot $\mathrm{A}: \mathrm{NF}-\mathrm{\kappa B}$, stem cells and breast cancer: The links get stronger. Breast Cancer Res 13: 214, 2011.

34. Fan Y, Mao R and Yang J: NF- $\kappa$ B and STAT3 signaling pathways collaboratively link inflammation to cancer. Protein Cell 4: 176-185, 2013

35. Thu YM, Su Y, Yang J, Splittgerber R, Na S, Boyd A, Mosse C, Simons $\mathrm{C}$ and Richmond $\mathrm{A}: \mathrm{NF}-\kappa \mathrm{B}$ inducing kinase (NIK) modulates melanoma tumorigenesis by regulating expression of pro-survival factors through the $\beta$-catenin pathway. Oncogene 31: 2580-2592, 2012.

36. Nakayama H, Ikebe T, Beppu M and Shirasuna K: High expression levels of nuclear factor kappa-B, ІкB kinase $\alpha$ and Akt kinase in squamous cell carcinoma of the oral cavity. Cancer 92: 3037-3044, 2001.

37. Arnott CH, Scott KA, Moore RJ, Hewer A, Phillips DH, Parker P, Balkwill FR and Owens DM: Tumour necrosis factor-alpha mediates tumour promotion via a PKC alpha- and AP-1-dependent pathway. Oncogene 21: 4728-4738, 2002.

38. Rhodus NL, Ho V, Miller CS, Myers S and Ondrey F: NF-kappaB dependent cytokine levels in saliva of patients with oral preneoplastic lesions and oral squamous cell carcinoma. Cancer Detect Prev 29: 42-45, 2005.

39. Senthilnathan P, Padmavathi R, Magesh V and Sakthisekaran D: Modulation of TCA cycle enzymes and electron transport chain systems in experimental lung cancer. Life Sci 78: 1010-1014, 2006.

40. Selak MA, Armour SM, MacKenzie ED, Boulahbel H Watson DG, Mansfield KD, Pan Y, Simon MC, Thompson CB and Gottlieb E: Succinate links TCA cycle dysfunction to oncogenesis by inhibiting HIF-alpha prolyl hydroxylase. Cancer Cell 7: 77-85, 2005.

41. Bayley JP, Devilee P and Taschner PE: The SDH mutation database: An online resource for succinate dehydrogenase sequence variants involved in pheochromocytoma, paraganglioma and mitochondrial complex II deficiency. BMC Med Genet 6: 39, 2005.

42. Hao HX, Khalimonchuk O, Schraders M, Dephoure N, Bayley JP, Kunst H, Devilee P, Cremers CW, Schiffman JD, Bentz BG, et al: SDH5, a gene required for flavination of succinate dehydrogenase, is mutated in paraganglioma. Science 325: 1139-1142, 2009.

43. Ricketts C, Woodward ER, Killick P, Morris MR, Astuti D, Latif $\mathrm{F}$ and Maher ER: Germline SDHB mutations and familial renal cell carcinoma. J Natl Cancer Inst 100: 1260-1262, 2008.

44. Janeway KA, Kim SY, Lodish M, Nosé V, Rustin P, Gaal J, Dahia PL, Liegl B, Ball ER, Raygada M, et al; NIH Pediatric and Wild-Type GIST Clinic: Defects in succinate dehydrogenase in gastrointestinal stromal tumors lacking KIT and PDGFRA mutations. Proc Natl Acad Sci USA 108: 314-318, 2011.

45. Raimundo N, Baysal BE and Shadel GS: Revisiting the TCA cycle: Signaling to tumor formation. Trends Mol Med 17: 641-649, 2011. 